\title{
SELF RUNTIME ENVIRONMENT USING ANDROID
}

\author{
Anikethan Shetty ${ }^{1}$, Moinuddin Pathan ${ }^{2}$, Shahrukh Khan ${ }^{3}$, M.Lutful Islam ${ }^{4}$ \\ ${ }^{1}$ Student, Computer Engineering, MHSSCOE, Maharashtra, India \\ ${ }^{2}$ Student, Computer Engineering, MHSSCOE, Maharashtra, India \\ ${ }^{3}$ Student, Computer Engineering, MHSSCOE, Maharashtra, India \\ ${ }^{4}$ Assistance Professor, Computer Engineering, MHSSCOE, Maharashtra, India
}

\begin{abstract}
Mobile devices influence our daily routine. The demand of mobile applications and the use of collaborative web systems are growing. Mobile devices are quickly becoming more popular in our day to day life. In education, m-learning has been an interesting topic for several years. The quantitative and qualitative increase in mobile devices that reach the average user opens more and more topics for research. Mobile devices powerful enough to run personal computers with the advancement of wireless and mobile technology. Learning with the help of mobile devices is a new approach towards education because it offers learning opportunities anywhere and anytime. Android Collaborative Learning using android operating system on mobile devices has been getting more importance in educational environment as one type of mobile learning application. This project introduces the designing and developing an effective self-learning environment for students as well as describing a new approach for building a learning application towards mobile technology.
\end{abstract}

Key words: m-learning, Android operating system, Android Collaborative Learning.

\section{INTRODUCTION}

Implementing a collaborative learning system using mobile devices requires technical and cognitive knowledge. Developers have to consider the usability of the mobile application and the collaborative environment to ensure effective learning activities. Use of mobile devices has steadily grown in popularity to become one of the most common consumer devices. Mobile devices are cheaper in comparison to laptop computers so that we can carry and use them anywhere in the world. Although the technologies have also been changing rapidly the educational environment and the programs of colleges/universities since new technologies propose different learning skill. Mobile applications are more flexible and can integrate the existing services by employing web-based interfaces. There are so many attractive learning tools have been designed and developed by integrating with such emerging technologies as motion sensor, Camera, Global positioning system (GPS), Wi-Fi, Bluetooth etc. Many attractive tools and techniques will be continuously integrated for creating an effective learning environment in the future.

Android Collaborative learning is based on the idea that learning is a naturally social act in which the learners talk among themselves. In today's life wireless technology has gaining more popularity amongst several users and therefore the mobile devices are reaching all levels of our educational learning levels. It is becoming a main communication since it is well suited in engaging collaborative learning environments. The concept of Android collaborative learning using android mobile devices is completely different from classroom-based learning. This way of the learning provides many possibilities such as providing opportunities to the group of people, working in same or different organizations to participate in accomplishment of specific goal using mobile devices.

\section{EXISTING SYSTEMS}

So far several mobile learning systems have been developed and there are other ongoing projects being carried out. As part of last autumn specialization project we reviewed similar solutions embarked upon by others; namely FABULA, MOBILEARN, Mobile Learning System for Scaffolding Bird Watching Learning and Mobile Computer Supported Collaborative Learning FABULA is a project that is being carried out by Software Engineering group and the Intelligent Systems group of the department of Information and Computer Science of the Norwegian University of Science and Technology (NTNU)

Mobile system for learning enabled by seamless roaming in mobile networks with its focus on services that foster the city learning geographies and ecologies [1] [2].

MOBILEARN is a worldwide European-lead research and development project that explores context sensitive approaches to informal, problem-based and work place. It is 
a project that aims at improving access to knowledge for selected target users such as mobile workers and learning citizens using new ways of mobile environments to meet the needs of the learners [1][3].

Mobile Learning System for Scaffolding Bird Watching Learning is a mobile system designed in Taiwan. It provides structured-assistance learning to users based on user's level of learning [1][4].

Mobile Computer Supported Collaborative Learning (MCSCL) uses mobile ad-hoc network, it aids students to collaborate in groups, monitoring real time progress regarding learning objectives and controlling the interaction, negotiation, coordination and communication [1][5].

A similar existing solution that was not reviewed last autumn but formed the basis of this project is Mobile Student Information System (MSIS). The MSIS system architecture is based on three tier architecture [6].

The MSIS application consist of three major services namely Location Search, Lecture Schedule Planner and Announcement. Location Search basically allows users to search for places such as lecture rooms, computer labs, canteens, etc.

Lecture Schedule Planner enables users to view information about course lectures and exercise guidance hours. Announcement provides news, notifications and other relevant information to the user. The location service implemented in the prototype of MCLS is an extension of the Location Search service provided in the MSIS application by Moe [6]

\section{ANDROID OPERATING SYSTEM}

Android is a simple mobile Operating System created by Google to run on any Electronic devices such as smart phones, e-books, Media Internet Devices (MID), MP4 players, notebooks and Internet tablets. Open and free hence most of the Android code was released under the opensource Apache License. The Main sladvantage of adopting android is that it offers a unified approach to application development. Developers need only develop for Android and their applications should be able to run on numerous different devices, as long as the devices are powered using Android. The Android Open Source Project (AOSP) is tasked with the maintenance and further development of Android. Android consists of a kernel based on the Linux kernel, with middleware, libraries and APIs written in $\mathrm{C}$ and application software running on an application framework which includes Java-compatible libraries based on Apache Harmony. Android uses the Dalvik virtual machine with just-in-time compilation to run compiled Java code. Android has a large community of developers writing applications ("apps") that extend the functionality of the devices. Developers write primarily in a customized version of Java. Apps can be downloaded from third-party sites or through online stores such as Android Market, the app store run by Google. Android uses SQLite a lightweight relational database for data storage. Android runtime includes the Dalvik Virtual Machine which enables every Android application to run in its own process, with its own instances of the Dalvik Virtual Machine (Android applications are compiled into Dalvik executables). Dalvik is a specialized virtual machine designed especially for Android and optimized for battery-powered mobile devices with limited memory and CPU.

\section{PROPOSED SYSTEM}

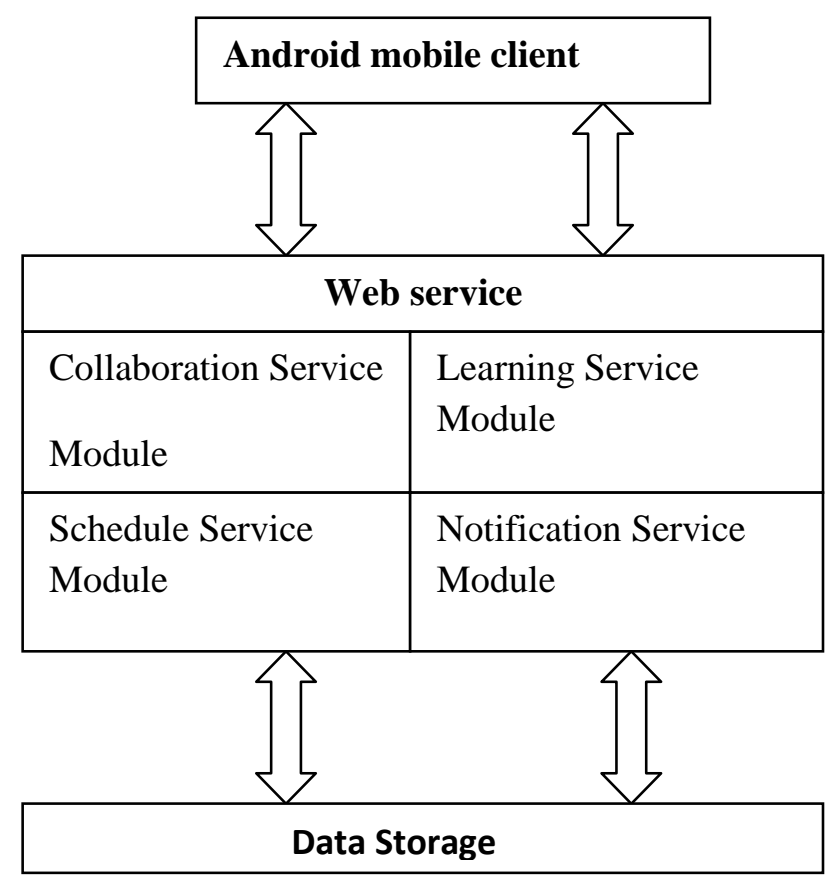

\section{ACL CLIENT APPLICATION}

The ACL client application is an application developed for Android based mobile devices. The client application provides the end-user with a graphical user interface which access services provided by ACL. The client application consists of fewer resources with non-critical data and functions processed on the client whilst all critical functions and processor intensive calculations are made on the external application server.

\section{ACL WEB SERVICES}

The ACL web services are application logic responsible for the processing and calculations of the functionalities provided by the system. The web services are implemented 
using ASP.NET . The web services use $\mathrm{xml}$ to code and decode data and SOAP to transport the data. SOAP has become a de-factor standard for web service communication, it is compatible with many different platforms and operating systems and enables machine to machine communication in very heterogeneous environments [7].There are two new web services in addition to two existing web services developed by Moe [6].The two new web service are Collaboration Service.asmx. and Learning WebServices.asmx. The other two existing web services used are UserService.asmx and LocationService.asmx.

\section{Collaboration Service}

This web service provides the functionality for sending and receiving messages, creating and joining groups. The Collaboration Service web service has the following methods:

SendGroupMessage( ): This method has GroupMessage object, Gid and sendername as its parameter. Gid specifies the group id and the sendername specifies the name of the user who sent the message.

The method returns true if the group message is sent successfully and return false if it fails to send the group message.

GetGroupMessage( ): This method has GroupId as its parameter. The GroupId specifies the id of the group, the method queries the database for group messages matching the group id specified and returns all messages belonging to that group.

GetUserGroups( ): This method has UserId as its parameter. The UserId specifies the id of the user. This method returns all groups a user belongs to.

SendPersonalMessage( ): This method has msg, uname and sender as its parameters. The msg is the message composed by the user, uname specifies the name of the recipient of the message and sender specifies the name of the user who composed the message. This method returns true when the personal message is sent and returns false if it fails to send the personal message.

GetPersonalMessage( ): This method has UserId as its parameter. It queries the database for messages matching id of a user and returns all messages sent to that user.

AddGroup(): This method can be used to create new groups. It has groupname and groupdescription as its parameters. The method returns true when a new group is successfully added to the database and returns false if it fails to add a new group to the database.

JoinGroup( ): This method has usname and gpname as its parameters. The usname specifies the username and the gpname specifies the name of the group. The method returns true if a user joins a group successfully and returns false if it fails.

\section{Learning Web Service}

The Learning Web Service is the web service responsible for the management of the learning resources. The Learning Web Service consists of the following methods:

GetAvailableResources( ): This method has a UserId as a parameter. It searches the database for the resources matching the id of a user and returns all the resources the user has.

AddResource( ): This method can be used to add learning resources to the database. The method has Resource object and url as its parameters with the url pointing to where the resource is located. The method returns true when a resource is successfully added and false if it fails to add a resource.

UpdateResource( ): This method can be used to update an existing resource. The method accepts Resource object and url. The method returns true upon successfully updating a resource and false if it fails to update a resource.

\section{OBJECTIVES}

Widening Participation: The key advantage for most regions was enabling wider access to ACL for "any person, anytime, anywhere".

Promoting Global Interactivity: Whether through ACL or in the classroom, regions highlighted the potential for increased communication between the teacher and the student as well as student-to-student communication.

Adapting and Flexibilizing teaching/learning: The use of digital materials and methods allowed easy adaptation and updating of material for an increasingly diverse student base, incorporating an ever wider range of teaching tools e.g. the capacity to provide content to specific student or question answer section. Transforming and inspiring potential of ACL for teacher and teaching method.

Quality and Efficiency: A final consideration was the potential of ACL to improve the efficiency and effectiveness of the learning process. 


\section{FROM THE STUDENTS END:}

- Students have to download an .apk file and installing that .apk file in his/ her android base device.

- Icon will create, after Clicking on the icon, Android edu system application will be started.

- Student can get the access to system by providing his/her username (email id) and password. Then Students can perform tasks like Events, Notification, Question \&Answer and Resources modules.

- Events: - Students get updates about college events and he/she can register for that event from a device.

- Notification: - Students will get updates about lectures status, like timing of the lecture, cancellation of the lecture etc.

- Question \& Answer: - Students will directly interact with the professor for any doubts regarding any subject. Also students can interact with each other on that topic (Group Discussion).

- Resources:-In this module student can directly upload the notes

Regarding any subject easily on the device and also access it anytime, anywhere.

\section{FROM THE ADMIN END:}

- Administrator has to launch the website on the server also providing link of the .apk file.

- Administrator first login for the site and accesses the site.

- Manage Student: - In this module admin will insert the students information on the site also can edit or delete that student's entry from the database.

- Manage Resources: - In this module admin can upload a notes on the site for the student reference.

- Manage Messages: - In this module Admin will broadcast the message about lectures status to the student.

- Manage Question: - In this module admin will answer to the student's queries.

- Manage Events :- In this module admin will broadcast the message related to any event in the college like workshop, college festival, Seminar,Freshers party etc. and also providing a facility for the registration to that event.

\section{ADVANTAGES}

- Easy to use, Synchronous collaboration.

- Provides user friendly interface.

- Users may need short start time for collaboration.

- Students should have access to check comments given by teachers regarding their performance and grades.

- Teacher should include critical notes for performance of each student after completion of ACL session and provide feedback to improve students in the future.

- To provide support for session management.

- To provide checking facilities to faculty to check group members.

- To provide freedom of thoughts to participating group members.

- Server should give messages of information updating.

- To provide support for users' role.

- Faculty should dedicate time to monitor the progress of participating members.

- To provide support to handle shared information.

- Communication should be based on broadcast with support of multicasting.

\section{CONCLUSIONS}

We have discussed the ACL user requirements develop a new ACL application to meet the requirements of students, teachers, teaching assistants, and administrators. However, the architecture will properly meet the current challenge of ACL and we will keep focusing on implementing the whole group application to meet new user needs and requirements .

\section{ACKNOWLEDGEMENT}

We are thankful to Mohammed Haji Saboo Siddik College of Engineering, for providing us the opportunity to do constructive work. We also thank anonymous reviewers for their constructive suggestions.

\section{REFERENCES}

[1] Noble Kuadey and Izaz U1 - Haq. Mobile Collaborative Learning System. Norwegian University of Science and Technology, Specialization Project Autumn 2009.

[2] FABULA, THE CITY AS AN ARENA FOR LEARNING, 
http://www.fabula.idi.ntnu.no/sites/default/files/FABULA_s hort.pdf retrieved 22.05.10 $\begin{array}{lr}\text { [3]MOBILEARN } & \begin{array}{r}\text { Project, } \\ \text { http://www.mobilearn.org/download/vision.pdf }\end{array}\end{array}$ 20.05.10

[4] Y.S. Chen, T.C. Kao \& J.P. Sheu; A mobile learning system for scaffolding bird watching learning; Journal of Computer Assisted Learning (2003) 19, 347-359 2003 Blackwell Publishing Ltd 347

[5] Zurita, G., Nussbaum, M., \& Shaples, M., (2003). Encouraging face-to-face collaborative learning through the use of handheld computers in the classroom. Mobile HCI 2003, Udine, Italy, Springer-Verlag, pp. 193-208

http://www.springerlink.com/content/vy2bu1nh8wk714wu/f ulltext.pdf retrieved 22.05.10

[6] Moe, S.P. Design and Evaluation of a User Centric Information Systems; Enhancing Student Life with Mobile Computing; Norwegian University of Science and Technology, 2009 Master Thesis

[7] Ballinger Keith (2003). .NET Web Services: Architecture and Implementation. Addison Wesley.

[8] http://developer.android.com/index.html.

\section{BIOGRAPHIES}
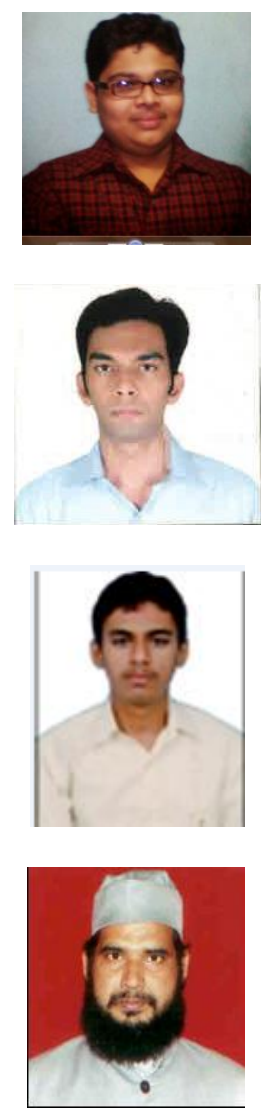

Name: Mr.Anikethan Shetty

Designation: Student

Department: Computer Engineering

Qualifications:B.E(comp)Pursuing

Name: Mr.Moinuddin M.Pathan

Designation: Student

Department: Computer Engineering

Qualifications:B.E(Comp)Pursuing

Name: Mr.Shahrukh J.Khan

Designation: Student

Department: Computer Engineering

Qualifications:B.E(Comp)Pursuing

Name: Mr. Md.Lutful Islam

Designation: Assistant Professor

Department: Computer Engineering

Qualifications:M.C.A,M.Tech(Comp) 